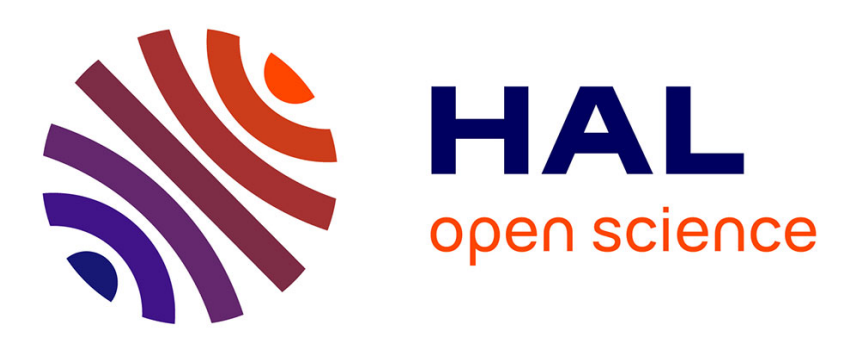

\title{
A novel approach on multi-shielded coaxial cable immunity assessment based on electromagnetism simulation
}

Clement Pornin, Tan Phu Vuong, Pascal Xavier, Gilbert Angenieux

\section{To cite this version:}

Clement Pornin, Tan Phu Vuong, Pascal Xavier, Gilbert Angenieux. A novel approach on multishielded coaxial cable immunity assessment based on electromagnetism simulation. 2018 Asia-Pacific Microwave Conference (APMC 2018), Nov 2018, Kyoto, Japan. pp.387-389. hal-02176175

\section{HAL Id: hal-02176175 \\ https://hal.science/hal-02176175}

Submitted on 7 Jul 2019

HAL is a multi-disciplinary open access archive for the deposit and dissemination of scientific research documents, whether they are published or not. The documents may come from teaching and research institutions in France or abroad, or from public or private research centers.
L'archive ouverte pluridisciplinaire HAL, est destinée au dépôt et à la diffusion de documents scientifiques de niveau recherche, publiés ou non, émanant des établissements d'enseignement et de recherche français ou étrangers, des laboratoires publics ou privés. 


\title{
A novel approach on multi-shielded coaxial cable immunity assessment based on electromagnetism simulation
}

\author{
Clement PORNIN* \\ , Tan Phu Vuong*, Pascal Xavier*, Gilbert Angenieux ${ }^{\dagger}$ \\ * IMEP - LaHC, GRENOBLE INP \\ 3 Parvis Louis Neel, 38000 GRENOBLE, France \\ clement.pornin@grenoble-inp.fr \\ tan-phu.vuong@grenoble-inp.fr \\ pascal.xavier@minatec.org \\ $\dagger$ IMEP - LaHC \\ Btiment "Le Chablais", Rue du Lac de la Thuile, 73370 Le Bourget-du-Lac, FRANCE \\ gilbert.angenieux@univ-smb.fr
}

\begin{abstract}
This paper introduces a novel approach for the assessment electromagnetic susceptibility of coaxial cables. In this work, a numerical model of the bulk current injection (BCI) fixture is considered. This fixture injects a current on the shield of the cable in order to produce electromagnetic interferences (EMI) and to extract the shielding features. An efficient and simple model is provided to avoid expensive prototyping steps. In addition, while the noise limits the interpretation of experimental data, the numerical modelling enables the consideration of a clear electromagnetic environment. Numerical and experimental results are compared for a sample of coaxial cable.

Index Terms-EMC; Cable shielding; cable susceptibility.
\end{abstract}

\section{INTRODUCTION}

Coaxial cables are widely used in wired communications for their shielding and mechanical properties. Since some sensitive systems are operating in compact electromagnetic environments, transmitting signals related to safety is a crucial task. Indeed, external spurious signals must be stopped by the shielding of the cable. This issue is even stronger when low intensity signals have to be transmitted. Thus coaxial cables with high electromagnetic immunity are used in hostile environments to ensures the signal integrity. These cables present several layers of heterogeneous shield, as defined in [1]. The design of this kind of cable is difficult. Indeed, the analytical solution describing the behaviour of the whole cable subject to electromagnetic interferences (EMI) is too complex to be described correctly. As an example, a mathematical model was developed in [2] to describe the behaviour of a ferromagnetic sheath of coaxial cables against EMI. The authors approximated the behaviour of one of the multiple layers of the shield we here considered. To assess their theory, a numerical model was used as a reference. Developing a mathematical model for a cable with several layers of shield would be hard, yielding to unacceptable uncertainties. The quality of the immunity of this kind of cable depends on many parameters as the structure, the dimensions and the material's electromagnetic properties. Thus, numerical modelling appears to be an efficient solution to remove prototyping steps and simplify the cable design.

In this paper, we propose :

- A numerical implementation of the Bulk Current Injection (BCI) fixture which aims to avoid to build prototypes usually required to assess a design. Additionally, this model is light and it can be ran on a personal computer.

- A solution that goes beyond the physical setup for numerical modelling can consider clear electromagnetic condition.

This paper is composed of two sections:

In section I, multi-shielded coaxial cables are introduced and their key feature is given. Then, a commonly used fixture to extract features of a cable architecture, based on BCI probes is introduced. The obtained results for two kinds of commercially available cables are shown as references. The first one is a single-shield coaxial and the second is a fivelayer-heterogeneous-shield coaxial cable. The limitation of the physical fixture is shown.

In section II, the electromagnetic model for Bulk Current Injection (BCI) fixture that uses finite elements method is introduced. The obtained results are presented for the architecture the single-shield reference cable used in the previous section. Then the comparison is used as a validation of the model.

\section{Multi-ShiElds COAXIAL CABLES AND MAIN FEATURE}

\section{A. Cable structure}

In the case of sensitive transmissions, multi-shields coaxial cables are used. This kind of cable is made of several heterogeneous layers of shield and combines the good conductivity of braided copper and high magnetic permeability of ferromagnetic layers (e.g. mumetal [3]). The copper layers aim at bringing a reference potential along the cable and insuring the current return. The ferromagnetic layers yield to trap the magnetic field in order to prevent direct coupling between copper layers. The main flaw of these cables comes from the connectors, where all the layers are electrically connected. 


\section{B. Transfer impedance measurement setup}

A common feature representative of the shielding efficiency of a wire is the transfer impedance introduced in [4]. The transfer impedance is defined by eq. 1, quantifying the difference of the potential $\Delta V[V]$ appearing between the shield and the ccore of the cable when a current $I_{s}[A]$ is conducted by the shield. $L[m]$ is the length of the considered cable.

$$
Z_{t}=\frac{\Delta V}{L \times I_{s}}\left[\Omega . m^{-1}\right]
$$

In [5], the authors present the triaxial method to measure $Z_{t}$. Triaxial method requires the insertion of the cable in a cylindrical conductor and forms a transmission line which takes the shielding of the coaxial cable as a core. A generator produces a $I_{s}$ current circulating on the shield. A $50 \Omega$ voltage probe measures $\Delta V$ at one end of the cable. The other end is short-circuited. This method is restrictive because it requires a rigid installation. This paper presents the bulk current injection (BCI) fixture, schematized in fig. 1, as a good alternative to the triaxial setup. Indeed, the BCI clamp injects the current on the shield, that is flexible, cheaper and more simple to set up than the triaxial method. Moreover, the electromagnetic environment remains the limitation of the physical setup, because the measurement of $\Delta V$ is noisy. This is especially true in the case of a very low transfer impedance, because $\Delta V$ is lower than the noise floor. Numerical modelling enables the use of a clear electromagnetic environment and permits the observation of the behaviour of the shield itself.

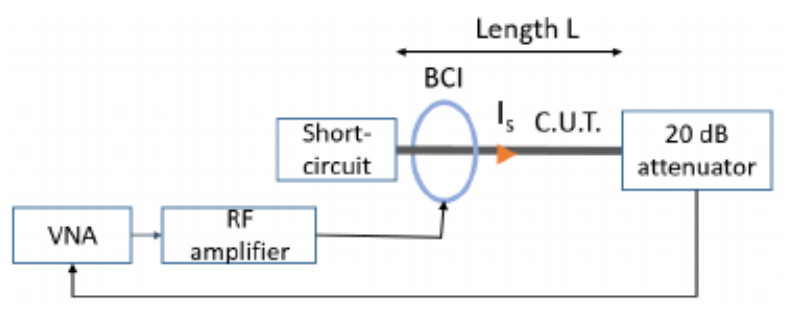

Fig. 1. BCI based setup for transfer impedance measurement

In order to directly display the transfer impedance at the VNA, a first measurement was made with a second BCI probe to display the $I_{s}$ current. The transmission parameter $S_{21}$ between the monitor probe and the injection probe was used to calibrate the vector network analyzer (VNA). Thus, the transfer impedance of the sample of cable was directly displayed at the VNA. Note that the length of the sample of cable was of one meter. Thus we did not need to consider the length to compute $Z_{t}$.

\section{Measurement on existing cables}

The transfer impedance of a standard RG-58 single-shield coaxial cable and a multi-shields, high-immunity coaxial cable was measured with the method introduced in the previous section. The results are shown in fig. 2 .

We can see that in the case of the single-shield coaxial cable, the the transfer impedance is smooth and varies in the

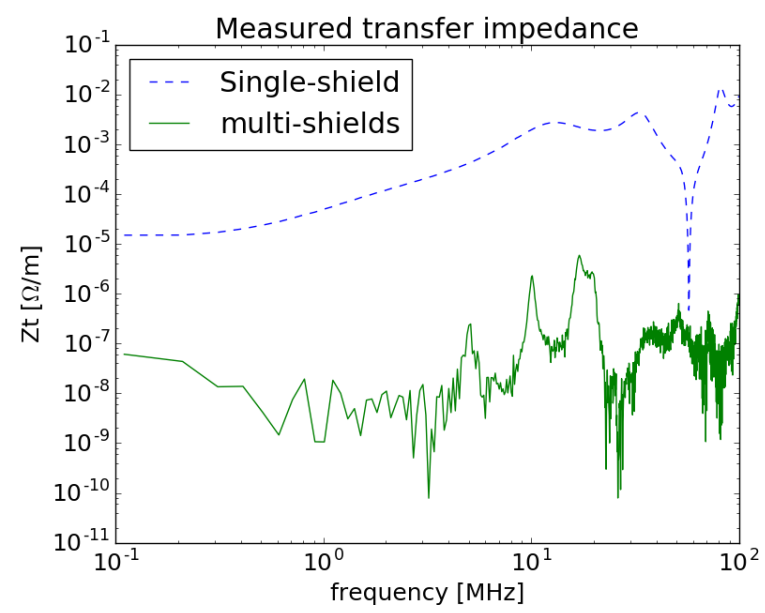

Fig. 2. Measured transfer impedances in the cases of one and five layers of shield

range $\left[10^{-2} ; 10^{-5}\right][\Omega / m]$. There is a minimum at $56[\mathrm{MHz}]$, caused by the stationary waves, depending on the length of the sample. In the case of the multi-shields coaxial cable, the measured transfer impedance is at a lower level in the range $\left[10^{-5} ; 10^{-10}\right][\Omega / m]$ and it is too noisy to give an accurate estimation of the cable features. This is the weakness of this method. The measure could be improved by using an anechoic room, which is an expensive structure and can not totally remove the noise. The numerical electromagnetic model introduced in the next section yields to pure electromagnetic (EM) environment and removes this limitation.

\section{BUILDING THE NUMERICAL MODEL OF THE FIXTURE}

\section{A. The BCI clamps}

The question of the BCI model has been widely treated in [6]. This paper gives instructions in order to get an electromagnetic model that exactly fits the behaviour of an existing instrument. We did not expect to obtain the exact behaviour of a specific clamp. Indeed, we wanted to use a model as plain as possible because we were interested in the behaviour of the cable itself. Our only requirement was to be able to inject a non-zero current in the range $[0.1 ; 100][\mathrm{MHz}]$. Thereby, we used CST Microweves studio (MWs) simulation software to build the BCI model model is shown in fig. 3 . This model is composed of a two torus. The first one, made of high magnetic permeability material, confines the magnetic field. The second torus, made of perfect electric conductor (PEC), is placed around the first torus. This torus is slotted and a discrete port is positioned at the slot level. The current conducted by the PEC creates a magnetic field that circulates in the other torus. This magnetic field induces a current in the inserted conductor, according to the Ampere's law.

Instead of using a monitor probe to measure the current $I_{s}$, a current monitor is positioned around the whole cable and another one around the central conductor. The value of $I_{s}$ is then given by the subtraction of both monitored current. 
The difference of the potential $\Delta V$ is obtained with a voltage monitor and the transfer impedance is computed using a postprocessing.

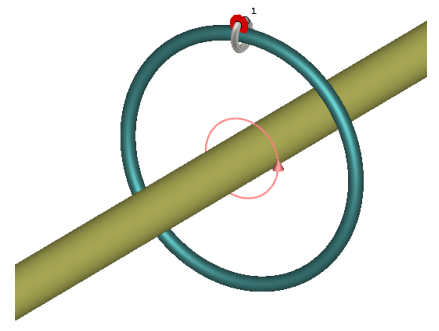

Fig. 3. Model of the BCI with a cable and shield-current monitor

\section{B. The cables}

The classic RG58 (see [7] for specifications) was considered in this work. The design of a simplified model of the cable, yielding to a fast computation, is allowed by the transmission line matrix (TLM) solver. This solver gives a specific mesh of the model by replacing thin layers by their analytical behaviours. Thereby, the total number of mesh cells is minimized. Nevertheless, for the sake of simplicity, braided layers were approximated as foil, which gives a different behaviour. The chosen thickness of the copper foil was $50[\mu \mathrm{m}]$ while the braid's wires diameter was $120[\mu \mathrm{m}]$. This choice was made by varying the thickness until obtaining a good agreement between plots of measurement and simulation.

\section{Simulation results and comparison to measurements}

The results obtained using the TLM solver for the RG58 cable model are shown in fig. 4 as well as the measurement with the BCI fixture, shown as a comparison.

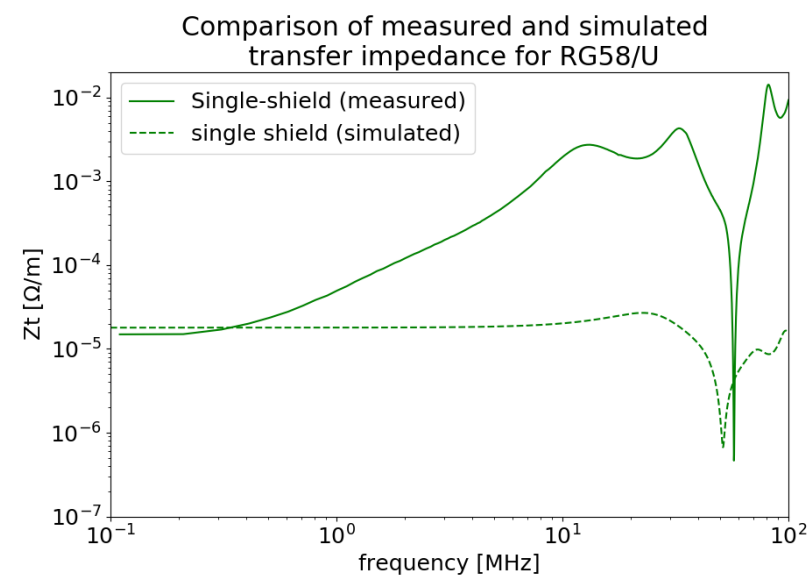

Fig. 4. Comparison between measured and simulated transfer impedances

We can see that while $Z_{t}$ increases with the frequency on measured data, the simulated transfer impedance stays flat. This behaviour is caused by the replacement of the braided shield by a copper foil. Foils are more efficient than braided shields due to several causes. The braided layers' transfer impedance depends on capacitive and inductive coupling through apertures [8]. On the other hand, skin effect is stronger in foils because the metal surface is less important than braids'. Nevertheless, for the sake of brevity, braided layers' model could not be introduced in this paper, even though CST MWs allows it. According to [9], the difference between simulation and measurement is due to the transfer inductance of the braid that makes frequency increases $Z_{t}$. The transfer inductance of the braid is used to model the coupling between braided conductors and diffraction through the structure's holes.

\section{CONCLUSION}

This paper introduces a simple way to obtain the shielding feature of a new cable design. This work has to be considered as a first step in order to build improved numerical models. Improvement can be done by considering the braided shield model. The novelty of this paper lies on the simplified injection method to subject cable design to EMI without being limited by the electromagnetic environment. Future work may introduce the particular behaviour of the braided layers, using an analytical solution to set the material directly with its surface impedance. Multi-shields cables will also be studied. The authors' goal is to help cable designers to lower the cost of this stage of the design.

\section{ACKNOWLEDGMENT}

The authors would like to express their gratitude to Chhayarith Heng Uy, Erika Vandelle and Edouard Rochefeuille for their technical support. This work was supported by the Region Rhone-Alpes-Auvergne grants.

\section{REFERENCES}

[1] J. A. Krabec, J. W. Kincaid, and P. B. Miller, "Multiply shielded coaxial cable with very low transfer impedance," US Patent US4 477693A, Oct., 1984. [Online]. Available: https://patents.google.com/patent/US4477693A/en

[2] D. E. Merewether, "Design of Shielded Cables Using Saturable Ferromagnetic Materials," IEEE Transactions on Electromagnetic Compatibility, vol. EMC-12, no. 3, pp. 138-141, Aug. 1970.

[3] "Mu Metal specifications include high and low magnetic permeability properties," Aug. 2018. [Online]. Available: https://www.mumetal.com/ mumetal_specifications.php

[4] S. A. Schelkunoff, "The Electromagnetic Theory of Coaxial Transmission Lines and Cylindrical Shields," Bell System Technical Journal, vol. 13, no. 4, pp. 532-579, Oct. 1934. [Online]. Available: https: //onlinelibrary.wiley.com/doi/abs/10.1002/j.1538-7305.1934.tb00679.x

[5] T. M. Benson, A. P. Duffy, and C. F. Cheng, "Assessing the performance of a current probe based method for determining surface transfer impedance," in 10th International Conference on Electromagnetic Compatibility, 1997. (Conf. Publ. No. 445), Sep. 1997, pp. 137-141.

[6] F. Grassi, "Accurate modeling of ferrite-core effects in probes for Bulk Current Injection," in 2009 IEEE International Conference on Microwaves, Communications, Antennas and Electronics Systems, Nov. 2009, pp. 1-6.

[7] V. Cheng, "specification_rg58u cable (2).pdf," Feb. 2005. [Online]. Available: http://www.rfcastle.com/download.php?file_path=data/goods/ specification_rg58u\%20cable.pdf\&file_name=specification_rg58u\% 20cable.pdf

[8] E. F. Vance, "Shielding Effectiveness of Braided-Wire Shields," IEEE Transactions on Electromagnetic Compatibility, vol. EMC-17, no. 2, pp. 71-77, May 1975.

[9] B. Demoulin, P. Duvinage, and P. Degauque, "Paramtres de transfert des cbles coaxiaux tresss et des discontinuits de blindage," Annales Des Tlcommunications, vol. 39, no. 9, pp. 433-445, Sep. 1984. [Online]. Available: https://doi.org/10.1007/BF03005182 